\title{
Density of Low-ionization Structures in PNe
}

\author{
Denise R. Gonçalves \\ Instituto de Astrofísica de Canarias, E-38205 La Laguna, Tenerife, \\ Spain
}

\author{
Romano L.M. Corradi \\ Isaac Newton Group - La Palma, Spain
}

Antonio Mampaso

Instituto de Astrofísica de Canarias

Cristina Campos

Departamento de Astrofísica, Universidad de La Laguna - Tenerife, Spain

\begin{abstract}
We have recently completed a comprehensive analysis of the morphological and kinematical properties of $\sim 50$ PNe which are known to possess low-ionization (micro)structures (LISs; Gonçalves et al. 2001). This study has shown that density is a crucial parameter for distinguishing among several physical processes responsible for their formation and evolution.

We are at present analysing long-slit, medium-resolution optical spectra of $18 \mathrm{PNe}$ in order to determine the physical parameters of the LISs and their environments. These data were obtained with the $1.5 \mathrm{~m}$ Danish (ESO) and the $2.5 \mathrm{~m}$ Isaac Newton (ORM) telescopes.

Here we show the first results of this survey, presenting the density variation of NGC 7009 and comparing it with the derived densities of K 4-47 and Wray 17-1.
\end{abstract}

\section{Introduction}

LISs contain important information about the mass-loss and radiative processes that lead to the formation and evolution of $\mathrm{PNe}$, and are identified as "smallscale" features of PNe that are especially prominent in low-ionization lines (commonly [NII]) and which have a size much smaller than the main morphological components of the PNe (like shells, lobes or haloes). We have studied - in terms of morphology and kinematics - a rich zoo of knots, filaments, jets and jet-like systems of low ionization, as well as compared their characteristics with the most promising physical processes for their formation, in previous papers (Corradi et al. 1999, 2000a,b; Gonçalves et al. 2001). 


\subsection{Preliminary Results for NGC $\mathbf{7 0 0 9}$}

NGC 7009 is a well known nebula (Balick et al. 1994, 1998) composed of a bright elliptical shell, as well as a pair of low-ionization high-velocity jets and a pair of knots. The tips of the jets, FLIERs, and the pairs of knots, lie in different directions (Reay \& Atlerthon 1985), being the tips of the higher ionized shell, the caps. The P.A. of our slit is $79^{\circ}$, allowing the simultaneous observation of all the above components. The density variation of NGC 7009 is shown in the table below. Densities were derived from the [SII] lines, assuming $T_{e}=10^{4} \mathrm{~K}$.

Table 1. Density variation of NGC 7009

\begin{tabular}{lcc}
\hline Region & Distance to the centre (') & Density $\left(\mathrm{cm}^{-3}\right)$ \\
\hline FLIER (East) & $21.7-26.6$ & 2025 \\
Jet (East) & $16.1-20.3$ & 850 \\
Cap (East) & $6.3-14.7$ & 4450 \\
Shell (East) & $1.4-4.9$ & 5075 \\
Shell (West) & $2.1-3.5$ & 5775 \\
Cap (West) & $4.9-14.0$ & 4975 \\
Jet (West) & $15.4-22.4$ & 1325 \\
FLIER (West) & $23.8-28.7$ & 1250 \\
\hline \hline
\end{tabular}

The typical density of the jets $\left(1000 \mathrm{~cm}^{-3}\right)$ is a factor of 4 or 5 lower than that of the shell $\left(5000 \mathrm{~cm}^{-3}\right)$. At the tips of the jets densities are a bit higher (FLIER-E) and a bit lower (FLIER-W) than their surroundings, the jet itself. On the other hand, both caps are less dense than the shell, the latter being the densest component of the nebula (typically $5400 \mathrm{~cm}^{-3}$ ).

K 4-47 has a pair of high-velocity jets that end in dense knots. At variance with the jets of NGC 7009, the entire inner region is significantly less dense than its tips. On the other hand, Wray 17-1 presents a low-velocity jet-like pair, in addition to a pair of low-velocity knots. Derived densities are roughly the same at the position of the LISs, as well as in the overall nebula.

Are the above results in agreement with the theoretical predictions of the density contrast of high-velocity jets? Is there a correlation between the kinematical state and the density of FLIERs, jets and jet-like systems? These and other discussions will appear soon in Gonçalves, et al. (in preparation).

\section{References}

Balick, B., et al., 1998, AJ, 116, 360

Balick, B., et al., 1994, ApJ, 424, 800

Corradi, R. M. L., et al., 2000a, ApJ, 542, 861

Corradi, R. M. L., et al., 2000b, ApJ, 535, 823

Corradi, R. M. L., et al., 1999, ApJ, 523, 721

Gonçalves, et al., 2001, ApJ, 547, 302

Reay, N. K., \& Atherton, D. P. 1985, MNRAS, 215, 233 